\title{
Ciencia y VAlores, OTRA VeZ: SOBRE LA INCORPORACIÓN DE VALORES NO EPISTÉMICOS EN LAS PRÁCTICAS CIENTÍFICAS
}

SCIENCE AND VALUES, ONCE AGAIN: ON THE INCORPORATION OF NON-EPISTEMIC VALUES IN SCIENTIFIC PRACTICES

\section{Alberto Oscar Cupani}

Universidade Federal de Santa Catarina, BRASIL cupani.alberto@gmail.com

\begin{abstract}
This article questions Matthew J. Brown's proposal to understand the relation between science and social values in regard mainly to two aspects: that the ideal of valuefree science should be abandoned; and that non-epistemic values can have a cognitive status. It retrieves nevertheless the emphasis given by Brown to the continually contingent character of inquiry and to the possibility of rationally resolving value conflicts, as well as the relevance of the social-pedagogic projects promoted by Brown.
\end{abstract}

Keywords: Value-free science $\bullet$ ethically responsible science $\bullet$ Matthew J. Brown

En un libro reciente, Matthew J. Brown retoma, con intención innovadora, el tema de la relación entre la ciencia y los valores (Brown 2020). ${ }^{1}$ Ese libro (Science and Moral Imagination. A New Ideal for Values in Science), en sus propias palabras, desafía la idea de que la ciencia debería ser "libre de valores", que califica de "mito". El autor sostiene que la ciencia es una práctica ética, que no debería estar distanciada, sino al servicio, de la sociedad, el medio ambiente y los derechos humanos. Cuestionando que la ciencia deba respeto sólo a la objetividad y la verdad, el autor alega que valores morales y sociales deben ser parte de ella. El libro aspira a orientar a los científicos y a las instituciones sobre la manera (correcta, admisible) de incorporar valores a sus prácticas. Brown no está, ciertamente solo en su cuestionamiento del ideal de valuefree science. Basta recordar, en las últimas décadas, contribuciones de autores como Helen Longino (1990 e 2002), Philip Kitcher (2001), Heather E. Douglas (2009), Kincaid e outros (2007) y sobre todo Hugh Lacey (1999, 2008, 2010, 2011).

Brown adopta, declaradamente, una posición pragmatista inspirada fundamentalmente en Dewey. Tratase - alega - de un "pragmatismo normativo", en un doble sentido: porque su abordaje de la normatividad es pragmático, y porque "el propio pragmatismo es tomado como un marco de referencia [framework] normativo de la 
práctica científica y de los juicios de valor" (Brown 2020, p.12). Supuesta esa perspectiva filosófica, Brown llama la atención para el hecho de que la práctica científica (y la ciencia es esencialmente eso para él) ${ }^{2}$ es siempre contingente, exigiendo a cada paso decisiones de los científicos, las cuales remiten a juicios de valor. Y "pocas, si es que alguna, de tales decisiones pueden, en principio ser decididas solamente por la lógica y las evidencias. Ni son suficientes los criterios epistémicos" (2020, p.19). Algún tipo de valoración está siempre en juego, epistémica o no epistémica, siendo que los valores epistémicos pueden ser influenciados por valores de otra naturaleza (morales, culturales) y que estos últimos pueden tener, ocasionalmente, un "status cognitivo" (una afirmación no intuitiva, que retomaré más adelante). ${ }^{3}$

El ideal de la ciencia libre de valores - observa Brown - estaba motivado por la presuposición de que los valores (en la forma de objetos de deseos e intereses) son siempre subjetivos, y que su admisión en la práctica científica sólo podía causar pérdida de la objetividad. Aquel ideal tampoco parecía diferenciar entre la valoración (valuing), que es generalmente irreflexiva, y el juicio de valor, que exige argumentación (la cual puede eventualmente ser demostrada como inválida). Ocurre que los "valores" (como referentes de nuestras necesidades, deseos, metas e ideales) no son forzosamente "subjetivos" - alega el autor - ni, consecuentemente, arbitrarios e imposibles de ser compartidos. Es necesaria, pues, una mejor teoría de los valores, y la que Brown propone ve los valores como "inherentemente conectados con la acción", provenientes de diversas fuentes, siendo de diversos tipos y desempeñando diversos roles en nuestra experiencia $\left(2020\right.$, p.20). ${ }^{4}$ Es igualmente necesario reconocer el carácter ético (sobre todo, en lo que se refiere a la responsabilidad) de la práctica científica y admitir que cuestiones relativas a valores pueden ser empíricamente discutidas y resueltas, pues se trata de problemas relativos a qué hacer en determinadas circunstancias (2020, p.20-21).

Establecer una analogía entre la investigación de cuestiones factuales y cuestiones axiológicas es una de las peculiaridades de la propuesta de Brown, quien también innova al asignar un papel relevante a la imaginación moral. Ese "ideal" es definido de la siguiente manera:

Los científicos deberían reconocer las contingencias en su trabajo como elecciones no forzadas [unforced], descubrir aspectos morales destacados de la situación que están decidiendo, reconocer y entender, mediante la empatía, a los legítimos afectados [stakeholders], reconstruir y explorar imaginariamente posibles opciones y formular juicios de valor justos y garantizados que guíen aquellas decisiones (Brown 2020, p.21, traducción mía, como todas las siguientes).

Ese ejercicio de imaginación tiene, según el autor, una triple función: asiste al científico cuando formula juicios de valor en la tomada de decisiones; limita las mismas al hacerlo consciente de sus implicaciones y consecuencias; y lo auxilia en la 
formación de ideales y metas que mejoren la ciencia y la vida humana. ${ }^{5}$ En todo caso, la importancia que le atribuye Brown se percibe cuando afirma que la falla de esa imaginación puede llegar a configurar una irresponsabilidad científica diferente de la mala conducta (deshonestidad) e igualmente reprobable (2020, p.22).

La propuesta de Brown sobre la relación entre la ciencia y los valores es ciertamente atractiva y en algunos aspectos avanza por comparación con las de otros autores, principalmente los anteriormente mencionados. Me propongo en este artículo examinar esa propuesta discutiendo, principalmente, dos de sus tesis: que el ideal de ciencia libre de valores no debe ser perseguido, y que los valores sociales tienen una función cognitiva.

\section{Sobre el ideal de ciencia libre de valores}

La noción, que se hace remontar como es sabido a Max Weber (Wissenschaft als Beruf, 1917), fue dominante en la Filosofía de la Ciencia reducida a reconstruir la "lógica de la investigación". ${ }^{6}$ Para Brown, la noción (y el ideal) de una ciencia ajena a valores reposaba en dos premisas: por un lado, la separación del "contexto de descubrimiento" y el "contexto de evaluación" de las teorías, y por otro, la convicción de la diferente naturaleza y papel de los valores epistémicos y no epistémicos (sociales, en resumen) (Brown 2020, p.62ss.). Mientras se admitía, como no problemática, la presencia de diversos tipos de valores en el contexto de descubrimiento, se rechazaba, como indebida, su intervención en el contexto de evaluación de las teorías. Aquí, los únicos valores legítimos eran los epistémicos (adecuación empírica, simplicidad, fecundidad, precisión, etc.), pues en la evaluación de las teorías, lo único relevante era la conexión lógica de las mismas con las evidencias. Cualquier otro tipo de valores (en particular, los éticos y los políticos) amenazaban el conocimiento producido en cuanto a su objetividad. ${ }^{7}$

Por detrás de la convicción de que valores no epistémicos tenían una influencia corruptora en la ciencia estaban — para Brown — dos estereotipos. El primero, que la ciencia alcanza un conocimiento "verdadero, cierto, confiable, inequívoco y decisivo" (Brown), por lo cual la actividad productora del mismo no podía ser contingente. ${ }^{8} \mathrm{El}$ segundo estereotipo era que los valores son puramente subjetivos, materia de preferencias personales, por lo que necesariamente constituían factores de distorsión del saber (bias). Pero esos estereotipos son, para el autor, falsos y perniciosos. El conocimiento científico no alcanza certezas, es sólo relativamente confiable, a menudo es equívoco y nunca es de por si decisivo (la decisión cabe a los hombres), para no mencionar el carácter problemático de la noción de verdad científica. Todo eso porque argumenta Brown - la práctica científica es, y no puede dejar de ser, contingente. No hay ningún recurso (tradición, método, principio) que vuelva necesario cualquier 
paso de la investigación, pues cada uno de ellos podría haber sido dado de manera diferente. La apariencia en contrario proviene del hábito y la confianza en la ciencia tal como viene siendo practicada.

En cuanto al segundo estereotipo - que los valores son siempre y puramente subjetivos - Brown observa que esa creencia es adoptada del sentido común e irreflexivamente. ${ }^{9}$ Pero los valores, sobre todo cuando se trata de juicios de valor, no se reducen forzosamente a lo que las personas prefieren o rechazan. Tales juicios son "decisiones reflexivas acerca de qué valorar, y son mejores o peores en base a razones" (2020, p.105), decisiones esas que pueden ser tomadas, según los casos, por individuos, equipos de investigación o la entera comunidad científica (2020, p.64). Aquellas razones, a su vez, pueden implicar cuestiones relativas a hechos, que pueden ser investigadas y resueltas de manera análoga a problemas científicos. ${ }^{10}$

Si yo afirmo - ejemplifica Brown — que el acceso a un razonable estándar de cuidado de la salud es un derecho de toda persona y usted lo niega, típicamente suponemos que hay aquí una cuestión legítima entre nosotros. No estoy [meramente] expresando una preferencia personal; en cambio, estoy haciendo una afirmación sobre el tipo de sociedad en que deberíamos vivir... Aunque esos desacuerdos pueden ser más difíciles de resolver que los desacuerdos sobre simples cuestiones sobre hechos, eso no les quita el sentido de que son genuinos desacuerdos" (2020, p.90).

Con todo, rechazar el subjetivismo axiológico no supone adoptar el objetivismo extremo, haciendo de los valores algo absoluto (como en la religión, o en ciertas doctrinas filosóficas). Tratase - para Brown — de entender los valores por su función en la vida, y en particular, en la investigación. Los valores son razones prácticas, razones para actuar (que, en una perspectiva pragmatista, son por lo tanto razones para creer o para afirmar). "Valorar [valuing] yace en el corazón de la acción, de la actividad, de la práctica. Valorar algo es estar dispuesto a actuar en favor de ello" (2020, p.15), y el entendimiento de los valores nos ayuda a dar sentido a nuestra acción. El origen de las valoraciones es múltiple (sobre todo, considerando la enorme variedad de las vidas individuales), pero ellas enraízan fundamentalmente, o en nuestra condición biológica, o en nuestra condición socio-cultural. Más específicamente, Brown distingue los siguientes tipos de valores (2020, p.121ss). Los biovalores (precondiciones de los otros), como la sobrevivencia y la adecuación al ambiente. Los valores sociales, como la comunicación, la cooperación, el cuidado, y la veracidad. .Los culturales (que indican metas e ideales comunes), como las costumbres y las maneras propias de la familia, el grupo o la clase, así como los criterios estéticos, las normas morales, las convicciones políticas y las creencias religiosas. ${ }^{11}$ Los valores personales, relativos a nuestra identidad y que derivan de "los compromisos que asumimos al tornarnos lo que somos", siendo particularmente importantes los valores profesionales (en este caso, los exigidos de los científicos). Existen también virtudes (valores) 
de instituciones, como la estabilidad y la justicia. Por lo demás, los valores pueden ser profesados consciente o inconscientemente. Y, naturalmente, las personas y las comunidades pueden variar en sus valoraciones a lo largo del tiempo. ${ }^{12}$

Aunque Brown no postula un valor como supremo, ni defiende cierto tipo de vida como mejor o preferible ("no existe una única forma de vida correcta", pues "hay una diversidad de razonables modos de vida", afirma en la p. 124), defiende a la democracia como la organización política que mejor permite la resolución de conflictos de valor, en materia de asuntos de interés público. Los valores inherentes a la democracia (entendida, siguiendo a Dewey, como una forma de cultura), tales como libertad, igualdad, solidaridad, publicidad y pluralismo, facilitan la transformación de las evaluaciones. Y aunque reconoce que la relación entre ciencia y democracia no es obvia, afirma que la cultura democrática posibilita que el conocimiento científico altere favorablemente los juicios de valor. ${ }^{13}$

Volviendo a la práctica científica, el caso es que, al resolver las constantes contingencias de la práctica científica, el recurso a valores (aunque sea implícitamente) se vuelve forzoso, según Brown. Eso porque "las diferentes opciones en esos momentos contingentes pueden tener implicaciones y consecuencias para cosas que nos importan" (2020, p.63). Juicios de valor, aunque sea irreflexivos, son usados para resolver las contingencias de la investigación, y pueden eventualmente ser cuestionados. Por lo tanto:

[Se muestra que] el ideal de libertad de valores no es solamente insostenible en la práctica, porque, por ejemplo, los seres humanos son limitados e incapaces de evitar completamente el pensamiento sesgado [biased] o motivado. Más bien, se muestra indigno [unworthy] e indeseable como ideal en principio. Los valores son penetrantes, inevitables y necesarios en la ciencia. La ciencia libre de valores no es ni siquiera deseable, si este argumento es correcto (Brown 2020, p.63, itálicos míos). ${ }^{14}$

\section{Sobre el papel cognitivo de valores no epistémicos}

Las afirmaciones anteriores de Brown deben ser completadas con sus ideas acerca del papel específico de los valores (epistémicos y no epistémicos) en la práctica científica, particularmente en su avance en la dirección de la ampliación del conocimiento. Para entender la posición del autor, hay que explicitar los reparos que formula a una confianza excesiva en los valores epistémicos y su crítica a una desconfianza no menos excesiva - a su juicio - con relación a los valores no epistémicos. Ese doble cuestionamiento está vinculado, como veremos, a un examen igualmente crítico de la noción de evidencia, central en cualquier reflexión sobre la ciencia.

Los "valores epistémicos" son, como ya recordé, aquellos criterios usados por los científicos para apreciar (o sea, juzgar) la confiabilidad de hipótesis, teorías, expli- 
caciones y resultados de la investigación. Criterios como la adecuación empírica, la coherencia (interna y externa), la simplicidad, la fecundidad y la precisión, que estamos acostumbrados a considerar como valores, sobre todo desde el famoso artículo en que Kuhn (Kuhn 1977) demostró que no se utilizan en la ciencia como reglas, sino como parámetros de apreciación variable. Brown critica la prioridad generalmente atribuida a esos valores, en el sentido de que las ideas científicas deben ser juzgadas fundamentalmente de acuerdo con ellos, apelándose sólo en segundo lugar a otras clases de valores. ${ }^{15}$ Ese privilegio - para nuestro autor - se vincula con la tesis de la "prioridad lexical de la evidencia", consistente en afirmar que la evidencia triunfa sobre cualquier otra consideración (contra factum non valet argumentum). Pero Brown señala que la noción de que un elemento de evidencia es un hecho particular (o su representación) es una noción superada (afirmación obviamente problemática) en la Filosofía da Ciencia, pues "muchas clases de cosas proporcionan evidencia en diferentes contextos" (2020, p.98). Por ejemplo, teorías pueden ser evidencia para otras teorías (como la de Kepler para la de Newton), así como conflictos con teorías bien establecidas pueden ser evidencia contra un descubrimiento. El argumento de la prioridad de los valores epistémicos reposa sobre la siguiente noción de una investigación bien conducida:

[...] resultados científicos ideales deberían consistir en un amplio cuerpo de evidencias fuertes, que sostiene inequívocamente una hipótesis o teoría internamente coherente, que satisface muy bien todos los estándares epistémicos y todos los valores relevantes, y que ha sido sometida a testeos rigurosos y debatida por una comunidad epistémica apropiadamente estructurada. (2020, p.94, itálicos míos).

"Todos los valores relevantes" puede aludir, por ejemplo, a no realizar (o no completar) una investigación que tenga connotaciones ética, social o ecológicamente reprobables. Sin embargo, añade Brown, generalmente debemos contentarnos con menos que eso. Por ejemplo, no raramente las evidencias no son inequívocas, o resultan difíciles de obtener en cantidad suficiente. O la teoría utilizada, a pesar de tener suficiente apoyo empírico, falla en resolver nuevos problemas de interés. Por otra parte, armonizar los valores/exigencias epistémicas con los otros tipos de valores y sus exigencias, puede no ser cosa fácil. Es claro que generalmente podemos insistir en perfeccionar nuestros instrumentos de investigación: encontrar nuevos datos, contextualizar la evidencia disponible, revisar nuestra teoría, etc. Pero hay situaciones en que no podemos esperar a agotar esos recursos epistémicos: tenemos que actuar, ${ }^{16}$ tenemos que legislar, tenemos que graduarnos, precisamos publicar... En tales casos, concluye Brown, es comprensible que los valores epistémicos no sean los únicos decisivos.

Por otra parte, también los valores epistémicos, si son defendidos de manera irrestricta, pueden conducir al wishful thinking, especialmente "cuando el asunto es com- 
plejo, cuando desafía las leyes de la lógica ordinaria, y cuando la evidencia tiende a despistarnos con relación a cómo el mundo realmente es" (2020, p.96). Eso, porque los estándares encierran suposiciones ontológicas o cosmológicas, como que el mundo, a un nivel fundamental, es simple y consistente, de tal modo que la aplicación tranquila de los estándares epistemológicos acaba reforzando esas suposiciones, tal vez inapropiadas.

En cuanto a la influencia de valores no epistémicos en la ciencia, ella es vista tradicionalmente como una amenaza, algo que interfiere en la búsqueda de la verdad o, en todo caso, como algo que no ayuda a la producción del conocimiento. Pero no deberíamos - alega Brown - priorizar la evidencia o los estándares epistémicos sobre otros valores de un modo generalizado. Esa es - argumenta - una posición infundada, vinculada a la creencia en el carácter puramente subjetivo de los valores y en su carencia de status cognitivo. Resulta que, ocasionalmente, valores no epistémicos pueden tener ese status. Los valores que pueden tenerlo no son los referentes del mero disfrute de las cosas o al de las actitudes tradicionales (que pueden encerrar prejuicios), sino los de los deseos, preferencias, anhelos y metas [goals]. En las ciencias biológicas, psicológicas y sociales, por ejemplo, conceptos como género, violencia o inteligencia (que son, nótese, descriptivos y valorativos al mismo tiempo) pueden funcionar como evidencias. (2020, p.99). Particularmente aptos para orientar la investigación considera Brown los ideales orientadores [guiding ideals], como el de que todos los ciudadanos tengan iguales derechos y oportunidades, y las cosmovisiones. ${ }^{17}$

Ese papel puede ayudar, o bien a dar verosimilitud a teorías que responden a esos valores, o bien a continuar buscando nuevas teorías cuando las disponibles contrarían aquellos valores. Brown menciona como prueba el carácter fecundo, reivindicado por las filósofas feministas, de sistemas de valores como el feminismo y el igualitarismo, así como su denuncia del perjuicio causado por el androcentrismo embutido en investigaciones consideradas correctas. Pero, a pesar de afirmar que, en caso de que juicios de valor tengan la misma función que la evidencia observacional o experimental deben ser considerados como hechos y no como valores, Brown limita la función de los mismos a establecer metas y limitaciones a la investigación (2020, p.143).

Ese papel cognitivo de valores no epistémicos no es, sin embargo, seguro. Que persigamos un objetivo - admite el autor - no garante que sea necesariamente valioso, y que una meta sea valiosa "nos da sólo una razón prima facie para dudar de conclusiones científicas que harían más difícil alcanzar esa meta" (2020, p.137). En caso de conflicto entre evidencias y valores - defiende Brown - tanto unas como otros pueden ser revisados. 


\section{Un ideal indeseable?}

Como se puede percibir, la discusión no gira en torno del hecho de que la ciencia esté o no comprometida con valores (que lo está es algo obvio, sobre todo con el advenimiento de la "tecnociencia"), sino de que la buena ciencia (correcta, honesta) deba responder a esa aspiración o deber, cumpliéndolo lo máximo posible. Tal aspiración fue consagrada por la Filosofía de la Ciencia reducida a radiografiar la "lógica de la investigación", ajena a discusiones sobre aspectos sociales e históricos de la práctica científica, ${ }^{18}$ y cuestionada por filósofos posteriores, sobre todo aquellosfamiliarizados con la historia y la sociología de la ciencia o interesados en ellas (como se volvió común después de los escritos de Kuhn y Feyerabend). Ese cuestionamiento alcanzó su ápice cuando diversos autores comenzaron a afirmar que el ideal de la value-free science era, no sólo irrealizable (en cualquier medida), sino también indeseable y hasta nocivo (Kitcher, Douglas).

Esa crítica estaba a menudo vinculada a la de la noción de ciencia "pura", "básica", supuestamente orientada a la "búsqueda de la verdad" o - menos pretensiosamente - a la ampliación del conocimiento confiable, sin propósitos prácticos inmediatos o previsibles. También, a la noción de la ciencia concebida como actividad individual de investigación, sin considerar necesariamente la dinámica de las comunidades científicas ni su relación con los contextos socio-culturales, excluyendo también otras actividades en que la ciencia participa, como la educación científica y la asesoría a grupos o instituciones. Hoy en día, la palabra ciencia evoca todas esas cosas, incluyendo su imagen mediática, y los filósofos no pueden ignorarlo.

Esa vinculación tiene como consecuencia la sospecha de que el ideal de ciencia sin valores pueda ser una meta anacrónica, aunque en principio fuera realizable. En ese caso, la apreciación de propuestas como la de Brown y otros autores (Kitcher, Douglas) podría ser aceptada con relativa facilidad. Bastaría admitir la complejidad de la ciencia, antes mencionada, y el carácter posiblemente ilusorio de la ciencia básica o "pura", ${ }_{19}$ para desembarazarnos de aquel ideal como de un espejismo maligno. Si a esa posición le añadimos la defensa de anhelos socioculturales caros a muchos intelectuales occidentales (como la defensa de los derechos humanos, la lucha contra la desigualdad social, la preocupación con el medio ambiente y el respeto a las diferencias culturales), está preparado el contexto para que una visión de la ciencia comprometida con valores (y que siga siendo "íntegra", como alega Douglas) se vuelva posible y hasta necesaria.

Ocurre, sin embargo, que la noción de ciencia básica (para evitar el polémico calificativo "pura"), o sea, la búsqueda del saber por su valor intrínseco, aunque filosóficamente difícil de defender, ${ }^{20}$ no deja de corresponder a cierta práctica (y numerosa). No es necesario disponer de datos estadísticos para suponer que hay muchos científicos dedicados, sí, a investigar problemas cuyo interés surge en un ámbito pu- 
ramente científico, a diferencia de los otros muchos que lo hacen por las aplicaciones que su solución tiene (ciencia "aplicada" y tecnología). Pero eso no exime a los primeros de ejercer la "imaginación moral" que pregona Brown (uno de sus aciertos, a mi ver), esto es, de preguntarse por las implicaciones y consecuencias de su investigación, más allá del contexto en que surgió el problema. Las investigaciones son financiadas (lo que obedece a cierta política, preferida a otras), posibilitan diversas aplicaciones (que pueden a su vez tener carácter moralmente cuestionable) y ocasionan modificaciones del mundo, natural y social, que pueden afectar negativamente a las personas, a las comunidades, a los seres vivos. Si el científico no piensa en todo ello (hasta donde lo puede hacer), incurre - vuelvo a darle razón a Brown - en una forma de negligencia moral. Esa exigencia vuelve ciertamente más difícil la práctica científica (puede implicar el abandono de la misma y hasta su denuncia), pero está a la altura de la importancia que nuestra época atribuye a la ciencia.

Lo anterior concierne a la decisión de dedicarse o no a un proyecto de investigación. ¿Qué decir de la tesis de Brown sobre el carácter constantemente contingente de la práctica científica, con la correspondiente necesidad de decidir y evaluar a cada paso? Pues que tiene razón. Su lente pragmatista le ayuda a destacar lo que es difícil negar, una vez percibido. La práctica científica (diferente de su "reconstrucción racional", que la idealiza) no responde a la pura lógica y el cálculo, sino que consiste en pasos que siempre podrían haber sido diferentes (una diferencia que se aprecia cuando hay brotes de originalidad o impases en la marcha de la ciencia), pero que son justificados por la tradición imperante. El papel del juicio de los científicos y las comunidades científicas para concluir (provisoriamente) disensiones es un asunto ya bien explorado (ver Cupani 2018, cap. 7). Por consiguiente, la eventual duda de un científico ante uno de aquellos pasos (el uso de cierta metodología, la confianza en determinado modelo, el peso de la evidencia disponible) es posible y legítima. Es también posible que su motivación para dudar no tenga base puramente epistémica (por ejemplo, al vacilar ante la aplicación de una técnica que parezca atentar contra derechos de las personas investigadas).

La ciencia básica, por lo tanto, no puede decirse ajena a valores no epistémicos. ¿Equivale esa admisión a condenar el ideal de ciencia libre de valores? Creo que debemos proceder con cautela, porque la condena de ese ideal está convirtiéndose en un lugar común (con todos sus riesgos), teniendo para muchos un estatuto "casi teológico" (G. Doppelt). ¿Qué queremos decir al afirmar que la ciencia debe (o no debe) ser "libre" de valores (no epistémicos)? Hay una comprensión tradicional: esos valores (convicciones morales, políticas, religiosas; intereses económicos y tecnológicos) no deben pre-determinar el resultado de una investigación. ${ }^{21}$ No veo motivos por los que esa comprensión precise ser abandonada (ni Brown lo sugiere). Hacerlo significaría igualar la ciencia a una ideología en el sentido que el autor utiliza la palabra. Está comprometida aquí la autonomía de la ciencia, en sentido etimológico de poseer su 
propia legalidad. Eso no significa, a mi ver, que ella sea independiente de su contexto social (cosa imposible), ni que consideraciones extra-científicas no puedan afectarla, sino que criterios no epistémicos, no son pertinentes, no vienen al caso, al decidir si una determinada conclusión científica está justificada. ${ }^{22}$ Sé que esta posición puede ser objetada alegándose que los valores epistémicos son contextuales e históricos. Ello es verdad en lo que se refiere a la amplitud del repertorio, o al peso dado a determinado criterio, pero no a la índole del propio repertorio. Hoy no se incluye la búsqueda de conocimiento cierto, a no ser en las ciencias formales, y la importancia atribuida a la precisión, por ejemplo, es variable; pero certeza (o probabilidad) y precisión son criterios que no se confunden con justicia, conveniencia, poder o sacralidad. ${ }^{23}$ Aunque los criterios epistémicos sean históricos, lo que cuenta es que en el momento de la investigación, esos (o sea, los criterios epistémicos tal como en la ocasión entendidos y admitidos) son los valores propios da ciencia. Cabe al científico (o al grupo) saber lidiar con la eventual influencia de valores extra-epistémicos de modo a que los epistémicos no sean sacrificados. Sugiero, en definitiva, que la libertad a la que alude el antiguo ideal se refiera a la autonomía de la ciencia, no a su (imposible) aislamiento con relación a valores (sociales, en resumen). ${ }^{24}$

Pero, según Brown, los valores son — como vimos — no sólo "penetrantes e inevitables", sino también "necesarios" a la ciencia bien practicada. Es decir, que el ideal cuestionado tendría el vicio de su supuesta virtud: la eximición axiológica. Precisamos de valores - para autores como Brown, Kitcher o Douglas - a fin de investigar correctamente. ${ }^{25}$ Debemos examinar ahora la alegación de que valores no epistémicos tengan una función cognitiva.

\section{Papel cognitivo de valores [sociales]?}

Para entender la posición de Brown a tal respecto, precisamos preguntarnos primeramente cuáles son los criterios epistémicos (o, más ampliamente, científicos) que él reconoce. Brown se refiere a lo que considera como "precondiciones de la ciencia" (ideales, más que requisitos mínimos), tales como la consistencia (no a cualquier costo), el control empírico, la sistematicidad (no reduccionista), y los "imperativos institucionales" del universalismo, el "comunalismo" y el "escepticismo organizado" de R. Merton (Merton 1942) ${ }^{26} \mathrm{~A}$ ellos agrega otros menos habituales como el compromiso pleno (whole-hartedness) con la investigación y sus resultados, el sometimiento a crítica (peer review) y el reconocimiento de crédito por la autoría (due credit). (2020, p.128-130). Sin adhesión a esos ideales, o "al menos su predominio", la ciencia no puede existir ni florecer. Pero advierte que no los considera universales ni absolutos (lo que configuraría una actitud "cientificista") pues su respeto es modificado por las circunstancias. Además, Brown sostiene que con el correr del tiempo la práctica 
científica en diversas áreas acabó consagrando "estándares epistémicos refinados", expresión con la que alude al repertorio de criterios habitualmente mencionados al abordar este tópico: simplicidad, amplitud de alcance, precisión, fecundidad. Son ellas - aclara - "virtudes" de teorías, modelos, explicaciones, a las que los científicos se refieren para justificar sus opciones. Pero "no son, por si mismos, criterios de ciencia buena [i.e. bien practicada]" y a veces están vinculados al contexto de alguna disciplina. ${ }^{27}$

La argumentación de Brown se basa, por lo tanto, en una concepción ampliada de los valores característicos de la ciencia, que excede el repertorio habitualmente considerado de "valores epistémicos". Eso es importante para comprender la atribución de "status cognitivo" a valores no epistémicos. Tal atribución tiene que ver con su cuestionamiento de la prioridad de la evidencia, ${ }^{28}$ se refiere - como vimos - a los juicios de valor relativos a ideales (sociales), limita aquel status al establecimiento de "metas y restricciones" (constraints), y tiene un carácter "sugestivo", no probatorio. Brown no es claro en lo que toca a esas "restricciones", pero se puede suponer que alude a no aceptar, eventualmente, una teoría, una explicación o un hecho científico, por más que parezcan bien fundados, cuando contrarían aquellos ideales (220, p.137).

La posición de Brown es (explícitamente) análoga a la de otros autores como Lacey, Longino, Kitcher y Douglas, en el sentido de defender que valores extra científicos pueden mejorar la práctica científica, no sólo volviéndola socialmente más responsable, sino también intrínsecamente más rigurosa. ${ }^{29}$ Cabe recordar que hace ya décadas que las filósofas feministas vienen denunciando investigaciones aparentemente correctas, pero imperceptiblemente distorsionadas por valoraciones implícitas. Igualmente importante ha sido la crítica de Stephen J. Gould (The Mismeasure of Man, 1996), que Brown reproduce en su Introducción, a la supuesta base científica del racismo. Esos ejemplos sugieren fuertemente que la defensa de ideales (en aquellos casos, de igualdad) puede, no sólo desenmascarar resultados científicos aparentemente sólidos, sino también orientar investigaciones (rigurosas) que ayuden a realizar los ideales defendidos. ¿Basta ese indicio para atribuir una función cognitiva a tales ideales/valores? Brown parece más confiado que aquellos otros autores (sobre todo, por su cuestionamiento del valor decisivo de las evidencias) en el poder heurístico, aunque así no lo llame, de los valores sociales en la ciencia. ${ }^{30}$

Los ideales pueden ciertamente tener una función heurística, semejante a la de ciertas hipótesis metafísicas y epistemológicas (que el mundo es ordenado y cognoscible; que tal o cual aspecto do mundo es más complejo de lo que se supone, y difícil de abordar por la ciencia estándar; etc.). ${ }^{31}$ Pero, tratándose de valoraciones (=de lo que debe, o no debe, ser), su utilización es más problemática. Es cierto que puede argumentarse que la investigación guiada por ideales que abrazamos (como el igualitarismo, la libertad, la democracia) tiene más chances de transformar la sociedad 
en esa dirección. Pero vale la conclusión inversa: ideales contrarios ayudan a producir ciencia que los favorece. Además, la realización de un determinado ideal no prueba su valor, a no ser para sus partidarios. Es casi inevitable que la realización de un ideal social sea, para los que no lo comparten, el resultado de una acomodación del mundo a una "ideología".

Con todo, es justo reconocer que Brown no es ciego para esos inconvenientes, al proponer que en casos de conflicto entre los resultados científicos y los valores, estos últimos también pueden ser revistos (revalorados, sustituidos, etc.). Brown ilustra esta última posibilidad con el ejemplo, imaginario, de un neurobiólogo que investiga el comportamiento de ratones con la finalidad de obtener resultados útiles en el tratamiento de enfermedades humanas (2020, p.156ss). La investigación incluye someter los animales a diversas privaciones y sufrimientos, y el neurobiólogo - que está impresionado por lo que la investigación revela sobre pensamientos y sentimientos de los animales - entra en crisis de conciencia a propósito de su trabajo. ¿Qué hacer? ¿Renunciar a su investigación y tratar de convencer a sus colegas para que lo imiten? ¿Tratar de sofocar la voz de su conciencia? Nuestro científico no sabe cómo resolver la tensión entre los beneficios esperados de su investigación y el bienestar de los animales. Brown sugiere que esta "paradigmática perplejidad moral" puede encontrar una salida al redefinir sus compromisos morales. ${ }^{32}$ ¿Hasta qué punto este tipo de investigación contribuirá realmente para mejores prácticas médicas? ¿Sería menos perturbador otro tipo de experimentos con los animales? ¿Cuál es el exacto significado de "bienestar animal"? El resultado puede ser que la investigación sin daños a los animales puede continuar, mientras que la dañosa sólo sería admisible cuando la probabilidad de beneficios médicos fuera muy alta (y tomando todo tipo de medidas para atenuar el sufrimiento animal).

Aún con esas aclaraciones y concesiones, parece excesivo hablar del status o de la función cognitiva de valores no epistémicos como lo hace Brown. Más convincente es la posición de G. Doppelt (Doppelt 2007, p.190), quien argumenta que los valores sociales pueden influenciar en la diferente concepción y aplicación de tres criterios/valores epistémicos: las clases de fenómenos que una ciencia debe explicar, predecir, etc.; las clases de teorías adecuadas para ello: y los tipos de razonamientos (deducción, inducción...) para establecer la validez de las teorías (lo que a su vez se proyectará en la clase de evidencia considerada relevante). Sólo por su influencia sobre aquellos criterios (y no de manera directa ni substituyéndolos) pueden los valores sociales afectar la ciencia. Igualmente preferible me parece la posición de Lacey, con su conocida distinción entre estrategias de investigación y las teorías a ellas correspondientes. Las estrategias están impulsadas por valores (como el lucro, el poder, o la salud, la justicia social), y condicionan las teorías consideradas adecuadas, pero estas últimas deben - para Lacey - ser propuestas y defendidas apelando exclusivamente a valores epistémicos ("imparcialidad" u "objetividad"). La existencia de 
estrategias impide que la ciencia sea neutra o neutral, mientras que la exigencia de imparcialidad garantiza que las teorías, sus explicaciones y aplicaciones no sean meros casos de defensa de una posición tomada. ${ }^{33}$ En definitiva, me parece que falta, en la propuesta de Brown, una explicación convincente de la manera en que valores sociales pueden afectar justificadamente los valores epistémicos (o, más ampliamente, los valores científicos que él admite) de manera que el resultado amplíe el potencial cognitivo de la ciencia al servicio de un ideal social.

Eso no quita méritos a su trabajo. Además de los que fui mencionando - como la importancia de la imaginación moral, el énfasis en el carácter contingente de la investigación y la posibilidad de resolver conflictos axiológicos - quisiera agregar que Brown hace bien en ampliar la noción de ciencia al analizar la relación de ella con valores. No sólo porque la ciencia es, hoy, una realidad omnipresente en la sociedad industrial, sino también porque aquella relación designa, en rigor, un conjunto de problemas que afectan la investigación, la aplicación, la difusión y la enseñanza de la ciencia. ${ }^{34}$ Otro mérito de Brown es su declarada intención de proporcionar guías para la práctica científica, intención respaldada por los proyectos pedagógicosociales que incentiva en sus estudiantes del Center for Values in Medicine, Science and Technology. ${ }^{35}$

La complejidad del tema "ciencia y valores" vuelve difícil proponer criterios o estrategias universales, esto es que sirvan para cualquier situación. Tal vez las soluciones deban ser siempre particulares (especialmente, en lo que respecta a cómo conciliar valores epistémicos y sociales), como o faz Lacey a propósito dos transgénicos (Lacey 2006). Y es posible que esas soluciones no puedan ser encontradas por filósofos en sus gabinetes, sino colaborando con científicos y otros profesionales (tecnólogos, políticos, pedagogos, etc.), sin desdeñar la opinión de personas comunes, en la medida en que la ciencia les ataña.

Por último, pero no por menos importante, es deseable que no se continúe utilizando la expresión "ciencia libre de valores", sea a favor o en contra. Está por demás claro que sólo suscita confusiones y polémicas, a veces inútiles. Es difícil sugerir otra que la substituya, pero "ciencia confiable" (J. Ziman) es una buena candidata. Griegos y troyanos pueden llenarla con sus respectivos criterios de confiabilidad.

\section{Referencias}

Brown, M. J. 2020. Science and Moral Imagination. A New Ideal for Values in Science. Pittsburgh: University of Pittsburgh Press.

Bunge, M. 1989 Treatise on Basic Philosophy. Vol. 8: Ethics: The Good and the Right. Dordrecht/Boston: D. Reidl.

Cupani, A. 2018 Sobre a Ciência. Estudos de Filosofia da Ciência. Florianópolis: Editora da UFSC. 
Dopplet, G. 2007. The Value Ladenness of Scientific Knowledge. In: Kincaid e outros, ValueFree Science? Ideals and Illusions, p.188ss.

Douglas, H. 2009. Science, Policy, and the Value-Free Ideal. Pittsburgh: University of Pittsburgh Press.

Kincaid, H.; Dupré, J.; Wylie, A. (org.) 2007. Value-Free Science? Ideals and Illusions. Oxford/New York: Oxford University Press.

Kitcher, P. 2001. Science, Truth and Democracy. Oxford/New York: Oxford University Press.

Kuhn, T. S. 1977. Objectivity, Value Judgment, and Theory Choice. In The Essential Tension: Selected Studies in Scientific Tradition and Change. Chicago: The University of Chicago Press, 320-339.

Lacey, H. 1999. Is Science Value-Free? London: Routledge.

Lacey, H. 2006. A controvérsia sobre os transgênicos. Aparecida (SP): Ed. Ideias e Letras.

Lacey, H. 2008. Valores e atividade científica 1. São Paulo: Ed. 34.

Lacey, H. 2010. Valores e atividade científica 2. São Paulo: Ed. 34.

Lacey, H. 2012. A imparcialidade da ciência e a responsabilidade dos cientistas. Revista Scientiae Studia, São Paulo, 9(3): 487-500.

Longino, H. 1990. Science as Social Knowledge. Values and Objectivity in Scientific Inquiry. Princeton: Princeton University Press.

Longino, H. 2002. The Fate of Knowledge. Princeton: Princeton University Press.

Merton, R. 1942. A ciência e a estrutura social democrática. (Science and democratic social structure) In: R. Merton. 2013 Ensaios de Sociologia da Ciência. São Paulo: Ed. 34, trad. De S. Gemingnani Garcia e P. R. Mariconda, pp.181-198.

Ziman, J. 2002 [2000] Real Science. What it is, and what it means. Cambridge: Cambridge University Press.

\section{Notas}

${ }^{1}$ Brown es especialista en Filosofía de la Ciencia, profesor de la Universidad de la Universidad de Texas en Dallas y director del Center for Values in Medicine, Science and Technology de aquella universidad.

${ }^{2}$ Esencial, pero no exclusivamente. Incluye en la categoría de ciencia no sólo la básica o "pura", sino también las investigaciones en ciencias tecnológicas y medicina. Además, afirma que "no ve diferencias significativas" entre las ciencias naturales como a las sociales en lo que hace a la cuestión aquí tratada (2020, p.16). Agrega que: "es por lo menos tendencioso tratar la 'ciencia pura' como si fuera la totalidad de la ciencia, o su caso central", pues "hasta la ciencia cultivada por sí misma tiene impactos externos sobre cosas que valoramos" (2020, p.75-76). Para Brown la "ciencia" abarca también la educación científica, el entrenamiento de los futuros investigadores, el asesoramiento [expert advising] y la búsqueda de subvenciones [grant writing] (2020, p.28-29)

${ }^{3}$ Es verdad que esas decisiones no son siempre conscientes, necesarias o posibles. Gran parte de la investigación científica se produce al abrigo de paradigmas que pre-deciden las opciones, de metodologías que las garanten o de plazos a cumplir que dificultan demoras. Pero la tesis de Brown apunta al hecho de que en principio tales decisiones "ocultas" pueden ser concientizadas, cuestionadas y juzgadas. (2020, p.59-60). 
${ }^{4}$ Esta conexión inherente de los valores con la acción es también sostenida por Lacey (v. Lacey 2008, cap. 2).

5 “En mi opinión, nuestra vocación ética y social más elevada es crear nuevos fines o metas, y esforzarnos por valores más complejos y una vida más intencional, en vez de vivir de manera habitual, sin pensar, o por algún propósito concebido remotamente a nosotros mismos" (Brown 2020, p.13).

${ }^{6}$ Eso no significa que los autores que defendieron esa concepción de la Filosofía de la Ciencia abrazaran ese ideal sin reservas. Ver Douglas 2009, cap.3.

${ }^{7}$ Douglas (2009, p.63-64) agrega la creencia en una separación de la ciencia con relación a la sociedad (autonomía).

${ }^{8} \mathrm{La}$ creencia en un método general de la ciencia habría funcionado como refuerzo de esa convicción. En vez de ese supuesto método, Brown sostiene - siempre desde su perspectiva pragmatista, para la cual la ciencia es "el arte de resolver problemas" - que existen pasos o fases (los que pueden ser recursivos) tales como: observación, encuadramiento del problema, sugestión (generación de hipótesis), razonamiento, prueba experimental y juicio (2020, p.3336).

${ }^{9}$ La mayoría de los filósofos de la ciencia, para Brown, asume una concepción emotivista o no cognitivista de los valores (2020, p.103).

${ }^{10}$ Este es otro de los aspectos innovadores de la propuesta de Brown, derivado de su posición pragmatista.

${ }^{11}$ Para Brown, reconocer el pluralismo de valores culturales no equivale a incurrir en un "craso relativismo" (en el sentido de que los juicios de valor son verdaderos relativamente a una cultura) sino asumir que los juicios que a ellos se refieren exige considerar el respectivo contexto (2020, p.124). El relativismo, además de suponer que las culturas existen aisladas unas de otras, imposibilita resolver conflictos de valor al impedir la comparación de valores.

${ }^{12}$ Sobre la índole de los valores, ver la exposición mucho más completa de Lacey (Lacey 1999, CAP. 2 e Lacey 2008, cap. 2). Vale recordar que Bunge se había adelantado a todos estos autores con su rigorosa y sistemática teoría de los valores en (Bunge 1989).

${ }^{13}$ Brown ilustra este punto con el caso de la valoración del hábito de fumar, que pasó de preferencia personal no discutible, a práctica censurable (inclusive, jurídicamente) con la divulgación de las investigaciones científicas que comprobaron su carácter perjudicial a la salud de fumantes y no fumantes (2020, p.133-34).

${ }^{14} \mathrm{El}$ argumento aludido es el de la contingencia de la investigación, calificado como "normativo" contra el ideal de ciencia libre de valores (2020, p.63).

${ }^{15}$ Por ejemplo, en los casos de "riesgos inductivos" o de "subdeterminación" de teorías, en que otros valores, como consideraciones éticas o creencias metafísicas pueden ayudar.

${ }^{16}$ Brown ejemplifica con el uso, en caso de emergencia, de vacunas no suficientemente testeadas durante una epidemia de ébola (2020, p.95, nota 10). El actual caso de la pandemia debida al coronavirus sería obviamente otro ejemplo.

${ }^{17}$ Brown las diferencia prudentemente de "ideologías", término por el que entiende aquellas convicciones que procuran acomodar las evidencias a sus previsiones, provocando "cerrazón mental" [closed-mindedness] (2020, p.141).

${ }^{18} \mathrm{~A}$ pesar de que Brown atribuye al Empirismo Lógico esa visión de la ciencia, afirmando que en la primera mitad del siglo XX tan sólo los marxistas y los pragmatistas admitían la relación de la ciencia con valores (2020, p.xiii), el panorama histórico es más complejo. Ver 
Douglas 2009: cap. 3.

19 "Pura" es una de esas palabras a la vez descriptivas y valorativas (como "mujer") sobre las que autores como Brown llaman la atención al denunciar la presencia de valoraciones ocultas.

${ }^{20}$ Kitcher (2001, cap.7) la considera un mito, al igual que Brown. Douglas (2009, p.76) cuestiona que el conocimiento pueda tener siempre prioridad sobre otros valores. Por otra parte, hasta un autor tan mesurado como John Ziman admitía que la noción del conocimiento buscado por sí mismo "desafía la lógica" (Ziman 2000, p.156).

${ }^{21}$ La analogía con el papel de las convicciones inherentes a un paradigma en la anticipación delo que ha de ser encontrado al abordar un problema de "ciencia normal" (Kuhn) no debe inducirnos a engaño. Se trata de cosas diferentes.

${ }^{22}$ Justificaciones a posteriori, como la eficacia de su aplicación, son tan sólo un refuerzo de las razones para creer en la confiabilidad de un resultado científico, que no la garantizan. (No soy pragmatista, A.C.)

${ }^{23}$ Esa separación es consecuencia de la diferenciación de las esferas culturales en la Modernidad, detectada por M. Weber.

${ }^{24} \mathrm{Me}$ opongo, por lo tanto, a entender la autonomía como aislamiento autoritario de la ciencia, sin responsabilidad con relación a la sociedad, como lo hace H. Douglas (Douglas 2009, p.7-8).

${ }^{25}$ Esa corrección no se refiere al mero cumplimiento de estandartes profesionales y la consiguiente obtención de conocimiento oficialmente confiable, sino a la producción de un saber que responda a ideales sociales, especialmente los democráticos (lo que es enfatizado en (Kitcher 2001).

${ }^{26} \mathrm{El}$ ethos de la ciencia, preservado por esos "imperativos institucionales" (R. Merton) es ciertamente un asunto problemático. Ver Cupani 2018, cap. 9).

${ }^{27}$ Brown menciona también convenciones como el estándar estadístico de $5 \%$ de margen de error , de uso común en diversas ciencias (2020, p.130)

${ }^{28}$ Brown se opone aquí, declaradamente, a autoras con propuestas similares, pero que no aceptan que valores puedan substituir evidencias (H. Longino, H. Douglas). Por lo demás, su defensa del "posible valor de evidencia" de los ideales/valores, es confusa (2020, p.137). Creo, en todo caso, que su ampliación de la noción de evidencia es inaceptable, pues equivale a transformar cualquier recurso en "evidencia".

${ }^{29}$ Brown aprecia las contribuciones de esos autores (as), pero las considera insatisfactorias en algunos aspectos. Llama la atención que no dialogue con los escritos de H. Lacey, cuyo tratamiento del tema es a mi ver más completo, aunque incluye en la bibliografía Is Science Value-Free? (1999).

${ }^{30}$ La confianza de Brown en ese papel de los valores no epistémicos lo lleva a defender, apoyándose en W. James (The Will to Believe) que en ciertos casos anhelos [desires] llevan a producir resultados deseados, principalmente cuando se trata de "fenómenos sociales que requieren acciones colectivas". En tales casos - concluye - "los valores no son evidencia, pero la preceden" (2020, p.100)

${ }^{31}$ La propia práctica científica de apoya en una hipótesis axiológica - que vale la pena investigar, en vez de meramente creer o aceptar - que parece ser confirmada por el éxito en el aumento de nuestro conocimiento.

${ }^{32}$ Esa salida no puede no resultar de la sola reflexión del investigador, sino que puede 
incluir discutir con colegas confiables, leer sobre ética de experimentación animal, consultar reglamentos y conocer prácticas de investigación consideradas correctas (2020, p.157). Para Brown, es crucial en ese examen determinar qué costo será admisible para alcanzar una finalidad.

${ }^{33}$ Lacey defiende también una noción normativa de la neutralidad de la ciencia, que consiste en permitir que diversas estrategias (y sus correspondientes compromisos de valor) sean practicadas respecto de un determinado asunto. Además, Lacey distingue entre "endosar" una teoría (adoptarla provisoriamente) y aceptarla definitivamente, otra útil distinción que puede ser aplicada a la propuesta de Brown de defender teorías que corresponden a determinados ideales (Lacey 2011).

${ }^{34} ¿$ Ha de continuarse a defender la superioridad irrestricta del saber científico frente al vulgar? ¿Cómo entender correctamente la autoridad social de los científicos? ¿Es posible delimitar claramente entre ciencia y pseudo-ciencia? ¿No es peligroso atribuir a la ciencia la revelación de la verdad sobre el mundo? Etc.

35 "Often, as the subject-matter allows, I stress social responsibility and public engagement through service learning projects in addition to traditional academic assignments. For instance, for my philosophy of technology and science, technology, and values courses, students have created projects aimed at disseminating information about sustainable technologies for rural communities in the developing world..." (de la página web de M. J. Brown, https://www.matthewjbrown.net/teaching, acceso el 14/03/2021). 